\title{
World Space Observatory-Ultraviolet: ISSIS, the imaging instrument
}

\author{
Ana I. Gómez de Castro ，Paola Sestito , Néstor Sánchez , Fátima López-Martínez \\ Juan Seijas , Maite Gómez , Pablo Rodríguez , José Quintana , Marcos Ubierna \\ Jacinto Muñoz
}

\begin{abstract}
The Imaging and Slitless Spectroscopy Instrument (ISSIS) will be flown as part of the science instrumentation in the World Space Observatory-Ultraviolet ( $W S O-U V$ ). ISSIS will be the first UV imager to operate in a high Earth orbit from a $2 \mathrm{~m}$ class space telescope. In this contribution, the science driving the ISSIS design and the main characteristics of this instrument are presented.

(C) 2013 COSPAR. Published by Elsevier Ltd. All rights reserved.
\end{abstract}

Keywords: Ultraviolet: General; Space vehicles: Instruments

\section{Introduction}

The resonance transitions of the most abundant species in the Universe as well as the electronic transitions of the most abundant molecules are in the ultraviolet (UV) range of the spectrum. Hence, access to the UV range is instrumental for progress in Astrophysics (Gómez de Castro et al., 2006). The World Space Observatory-Ultraviolet (WSO$U V$ ) space telescope will grant this access in the post-Hubble Space Telescope era. WSO-UV is a $170 \mathrm{~cm}$ diameter primary telescope with an efficient Ritchey-Chrétien configuration that will be operational in the 2017-2027 time frame. $W S O-U V$ is a Russian-led mission and will be equipped with instrumentation for UV spectroscopy and imaging (see Shustov et al., 2011).

The Imaging and Slitless Spectroscopy Instrument (ISSIS) will be a key part of the WSO-UV instrumentation. ISSIS is the first UV imager to be flown to high Earth orbit, above the Earth geocorona. Therefore, the UV background will be dominated by the zodiacal contribution and the diffuse galactic background due to dust-scattered starlight (Sujatha et al., 2009; Murthy et al., 2010). The instrument has been designed to make full benefit of the heritage left by the GALactic Evolution eXplorer (GALEX) mission. $G A L E X$ has surveyed about $80 \%$ of the sky at UV wavelengths, providing for the first time a nearly complete view of the UV Universe (Martin et al., 2003; Bianchi et al., 2011). However, GALEX spatial resolution was $\sim 4.2$ arcsec and had very moderate spectroscopic capabilities. ISSIS resolution will be $\leqslant 0.1$ arcsec. The Fine Guiding System of the $W S O-U V$ telescope will guarantee a very high pointing stability (better than $0.1 \operatorname{arcsec}$ at $3 \sigma$ ). Moreover, ISSIS will be equipped with gratings for slitless spectroscopy with spectral resolution 500 in the full $1150-3200 \AA$ spectral range. In imaging mode, the ISSIS effective area is about 10 times that of the GALEX imagers.

ISSIS is designed to be an instrument for analysis of weak UV point sources or clumpy extended sources, especially those with well defined geometry. UV imaging instruments have been often equipped with prisms or very low dispersion grisms. The rapid decay of the resolution of prisms, such as the available in the Solar Blind Channel of the Advanced Camera System, makes very difficult its use 
to map extended line emission at wavelengths above some $1350 \mathrm{~A}$. As the transmittance of narrow band filters in the far UV is $\leqslant 3 \%$, integral-field low-resolution spectroscopy is the main mean to map nebular emission. ISSIS gratings will make feasible to use the powerful UV diagnostic tools to determine the location of dusty blobs and measure electron densities and temperatures. In this contribution, the science that has driven to ISSIS design is described in Section 2. ISSIS design is presented in Section 3 and its performance is described in Section 4. ISSIS planning and a brief summary are provided in the concluding Section 5 .

\section{Science with ISSIS}

The key scientific drivers of the $W S O-U V$ project are:

- Galaxy formation: the evolution of the Star Formation Rate with redshift $(z)$ and the role of the intergalactic medium (IGM) as a mass supply for star formation at moderate redshifts $(z<2)$ - To study the characteristics of the diffuse baryonic content of the Universe and its chemical evolution; to determine, in particular, the baryonic content of the warm and hot IGM and of damped Lyman- $\alpha$ systems; and to investigate the role of starbursts in the chemical evolution of galaxies and the IGM.

- The formation and evolution of the Milky Way - To estimate the energy inputs of the gas interacting with stars; and to investigate the role of magnetic fields on star formation.

- The physics of accretion and outflows: the astronomical engines - To study stars, black holes, interacting binaries and, in general, all those objects where mass accretion has a relevant impact in the system evolution; and to analise the efficiency and time scales of these phenomena as well as the role of magnetic fields, radiation pressure and disk instabilities.

- Extrasolar planetary atmospheres and astrochemistry in presence of strong UV radiation fields - To measure the UV radiation field from $\mathrm{T}$ Tauri stars and its impact on the chemical evolution of young planetary disks and planetary atmospheres.

For this purpose, the $W S O-U V$ telescope is equipped with the imaging instrument ISSIS and the three WSO-UV Spectrographs (WUVS; Sachkov, 2010 and M. Sachkov, private communication, 10 May 2013):

- VUVES, the far-UV high-resolution echelle spectrograph operating in the $1115-1760 \AA$ range with resolution $\mathrm{R} \sim 50,000$.

- UVES, the near-UV high-resolution echelle spectrograph in the 1740-3100 $\AA$ range with resolution $\mathrm{R} \sim 50,000$.

- LSS, the Long-Slit Spectrograph (LSS) for low-resolution $(\mathrm{R} \sim 1000)$ long-slit spectroscopy. The width of the slit will be 1 arcsec and the spatial resolution 0.5 arcsec.
LSS and ISSIS provide together a powerful tool to study extended sources since the high resolution imaging of ISSIS complements the LSS field selection capability. Both ISSIS and LSS have similar spectral resolutions. The main topics that could be investigated with the instrument ISSIS cover a wide range of astronomical issues:

- Solar System - The atmospheres of the planets and satellites will be investigated as well as the impact of the Solar radiation in their evolution. The connection between the Solar System and exoplanetary systems, especially the magnetospheric processes in Jupiter and Saturn and the photoevaporative processes in comets, will be addressed.

- Stars in the Milky Way - The studies concerning our Galaxy will focus on various subjects: stellar clusters, for which an UV atlas will be constructed and information about proper motions and stellar evolution will be obtained; planetary nebulae, their proper motions, expansion and shocks, as well as the search for companions in infrared-bright asymptotic-giant-branch and proto-planetary nebulae (Guerrero et al., 2010, 2012); protostellar jets and the mechanisms at work in these interesting phenomena (Gómez de Castro and Robles, 1999; Coffey et al., 2004); the connection between magnetic activity and mass from solar-like stars to brown dwarfs and planets (Gómez de Castro et al., 2012); peculiar objects, such as some of the members of the $\sigma$ Orionis cluster, which will provide information about the complete spectrum of stellar masses in the UV from $O$ stars down to T Tauri stars and brown dwarfs (Caballero et al., 2006); absorption features in the stellar radiation by the atmospheres of transiting planets (Lecavelier des Etangs et al., 2004, 2010).

- Extragalactic astronomy - The ISSIS instrument will be able to observe efficiently extragalactic objects: studies of gravitationally lensed quasars will reveal the intrinsic variability of the nuclear continuum, as well as the effects on the lensing galaxy (Goicoechea and Shalyapin, 2010). Lyman- $\alpha$ emitters up to redshift $z \sim 2$ not visible from Earth can be investigated with the WSO-UV; Atlases of massive stars in galaxies of the Local Group will be built, together with a detailed inspection of their winds (García et al., 2011). High-mass X-ray binary systems and their environments will be monitored (Blay et al., 2009).

The ISSIS scientific requirements (Gómez de Castro et al., 2012a) driving the design are the following:

- High-resolution mapping of weak and nebulous sources such as microjets or gravitational lenses.

- Mapping of UV emission lines in extended emission nebulae (H II regions, supernovae remnants, planetary nebulae) and jets (from protostars or compact objects).

- Efficient spectroscopy of weak sources such as brown dwarfs, micro jets or star forming galaxies at low redshift $(0.5<z<1.5)$. 
- Resolution of at least $\mathrm{R} \sim 500$ to study the absorption of stellar radiation by transiting planets, or to derive the terminal velocity of winds from $\mathrm{O}$ stars in the Local Group.

- Enhancement of the dynamic range with coronographs or masks in order to map the faint emission close to bright sources (e.g. disks, jets, binary components).

- Short time resolution to track the evolution of instabilities in disks surrounding compact sources.

\section{ISSIS designs}

The instrument is located below the primary mirror and above the optical bench. This location imposes additional constraints to the design, in terms of weight and size: a maximum of $61.5 \mathrm{~kg}$ on the optical bench is allowed, and the full instrument has to be fit within a flat cylinder of height $17 \mathrm{~cm}$. ISSIS is fed by the central part of the beam but a pick-up mirror is required to fold the beam from the telescope adding one reflection (see Fig. 1). The final design is a compromise between the scientific requirements and the telescope/platform requirements (Gómez de Castro et al., 2011, 2012a,b).

Fig. 2 shows the layout of the instrument. ISSIS has two channels for imaging and slitless spectroscopy:
- The Far Ultraviolet (FUV) channel: working in the range $1150-1750 \AA$.

- The Near Ultraviolet (NUV) channel: covering wavelengths in the $1850-3200 \AA$ interval.

Both channels are equipped with photo-cathods with Micro Channel Plate (MCP) amplifiers and CMOS reading. CsI and CsTe photocathods have been selected for the FUV and NUV channels, respectively. The use of MCPs imposes strong constraints to the instrument capabilities, in particular on the field of view (FoV), barely 70 arcsec, and on the dynamical range. There are ongoing studies to increase the dynamical range by using masks. A set of neutral filters has been included to be able to satisfy the sensitivity requirements keeping the possibility to observe sources of moderate brightness. Slitless spectroscopy is not only an efficient mean to analyse multi-objects fields, but at the same time it acts as a narrow-band filtering technique.

The ISSIS layout is arranged as a telephoto system, where positive elements are followed by negative elements, to enlarge the overall focal length. A refocusing mechanism, located at the coupling stage between the telescope and ISSIS, changes the distance between the intermediate image from the telescope and the first mirror of ISSIS. The operation modes are imaging, spectroscopy, and
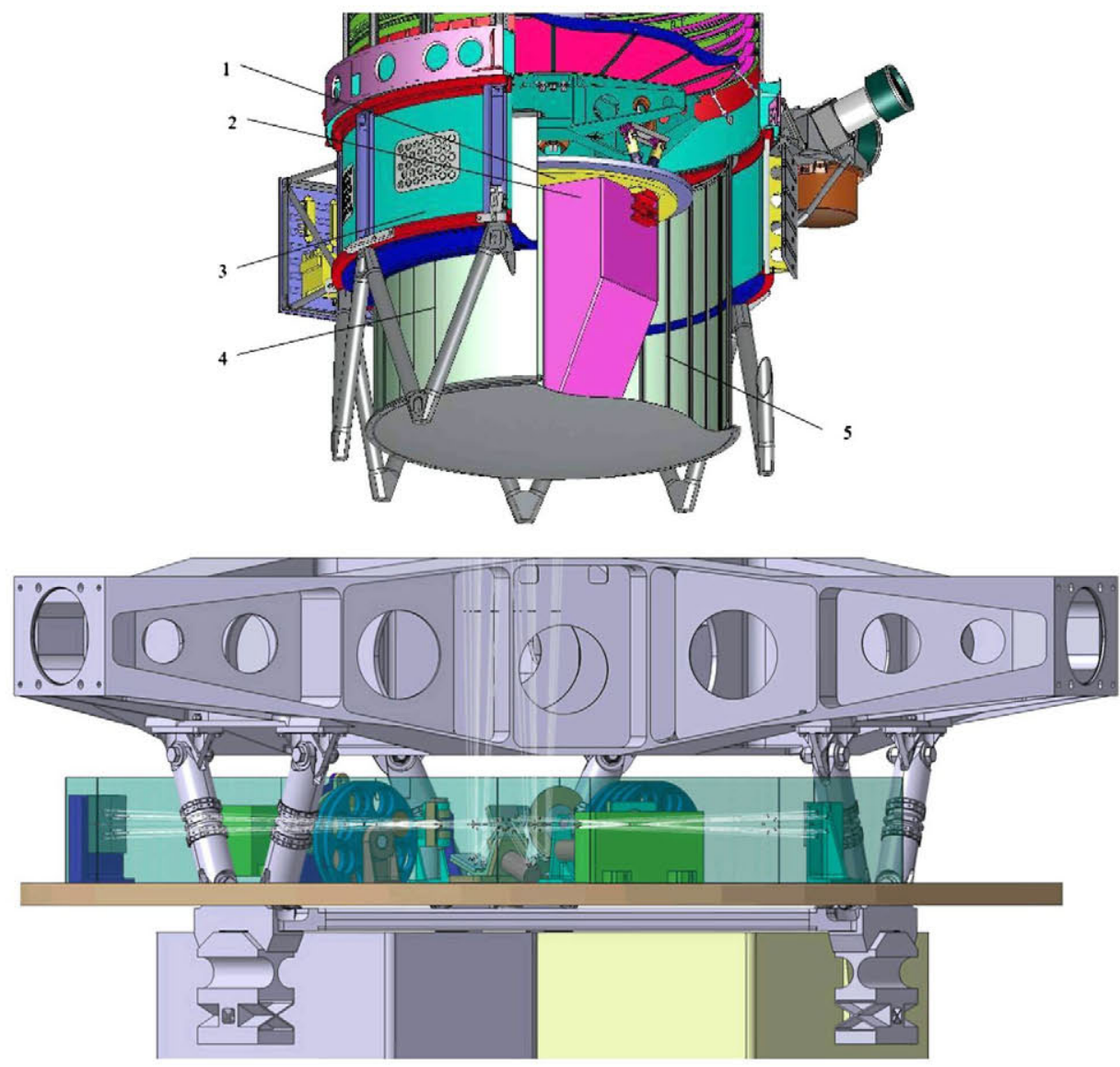

Fig. 1. Top: Instruments compartment in WSO-UV: (1) optical bench, (2) WUVS spectrographs, (3) external instrumentation panel, (4-5) lateral panels. ISSIS is above the optical bench and below the primary mirror. Bottom: Detail of the ISSIS location in the telescope. 


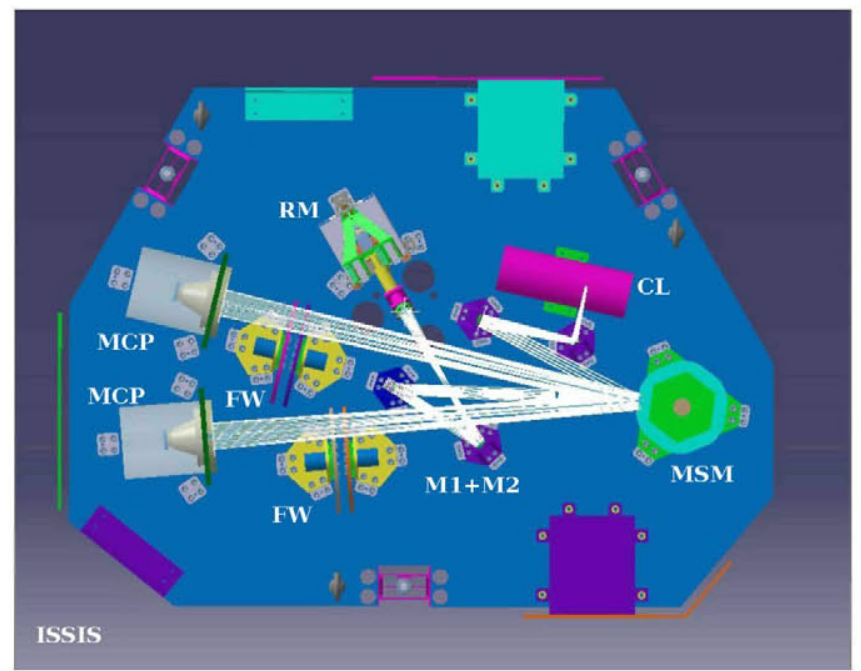

Fig. 2. Layout of the Imaging and Slitless Spectroscopy Instrument (ISSIS). The acronyms mark the location of detectors (MCP), filter wheels (FW), refocusing mechanism (RM) with pick up mirror, calibration lamp (CL), mode selector mechanism (MSM) and the mirrors M1 and M2.

calibration, and are selected through a mode selection mechanism (MSM; see Fig. 3):

- Imaging: the MSM uses flat mirrors aligned to direct the light into the selected channel (FUV or NUV). The optical filters are accommodated on two wheels for each channel; there are long-pass and narrow-band filters for specific investigations, as well as two neutral filters for each channel, to increase the dynamical range.

- Spectroscopy: the beam is directed to the dispersive elements, which are reflection gratings located at the MSM. In the nominal spectroscopy mode, the filter wheels are positioned into a hole configuration (no filter). The FUV diffraction grating has peak efficiency at $1400 \AA$ and groove density of 450 lines $/ \mathrm{mm}$, while the NUV one has the peak at $2300 \AA$ and 250 lines $/ \mathrm{mm}$.

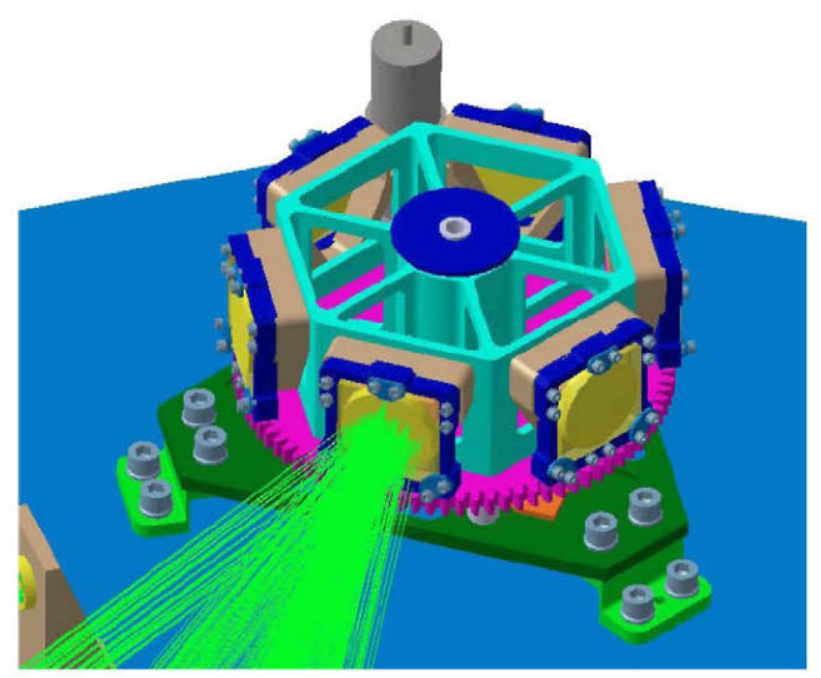

Fig. 3. Mode selection mechanism in ISSIS.
- Calibration: a shutter is used to block the light from the telescope, and the beam is directed to the calibration subsystem in order to take flat-field images on a pixelto-pixel basis. The calibration unit includes the lamp, optics and shutter.

\section{ISSIS performance}

The total throughput of ISSIS includes the reflectivity of the T-170M telescope and ISSIS mirrors, the diffraction grating reflectivity and the sensitivity curves of the MCP detectors. The expected value for the imaging mode will be of about $1 \%$ at $1300 \AA$ (FUV) and $7 \%$ at $2500 \AA$ (NUV), while for slitless spectroscopy the peaks will be about $0.4 \%$ at $1300 \AA$ and $3 \%$ at $2500 \AA$ (FUV and NUV, respectively). The radiometric sensitivity of ISSIS can be evaluated using the exposure time calculator (ETC) described below. Fig. 4 shows the throughput of the various components and the estimated total throughput in the whole wavelength range.

One of the ISSIS key drivers is the mapping of extended sources; this implies a proper sampling of the the point spread function (PSF). The Fine Guiding Sensors in the $W S O-U V$ should provide a spatial resolution of 0.1 arcsec at $3 \sigma$. Thus, the ISSIS optical system modifies the T-170M focal length to have this PSF sampled by 2-3 pixels. According to optical simulations, an asymmetrical PSF is expected: this effect cannot be avoided at UV wavelengths, since the optical quality is dominated by geometrical, nonsymmetric aberrations. The asymmetry in the PSF is close to $17 \%$ and to $8 \%$ in the FUV and NUV channels, respectively. Fig. 5 displays an example of PSF simulation on two different scales; since the two channels share the same optics, a similar PSF behaviour is expected for both of them.

The field of view is limited by the photocathods sensitive area in the MCP detectors and the spatial resolution to $\sim 1.2$ arcmin. This field of view will permit direct imaging as well as obtaining images of extended nebular objects in the most prominent spectral lines in the slitless spectroscopy mode. Table 1 shows a summary of the main ISSIS characteristics.

\subsection{Exposure time calculator}

Astronomers can use the ETC developed by the science team at Universidad Complutense de Madrid to evaluate the exposure time needed for an observation, in order to achieve a desired signal-to-noise ratio $(\mathrm{S} / \mathrm{N})$, and vice versa. The ETC is a web-based application available through the project website at http://www.wso-uv.es. It appears as a user-friendly interface with five main fields shown in Fig. 6, which are:

- Channel: to select among the two ISSIS channels (FUV and NUV). 

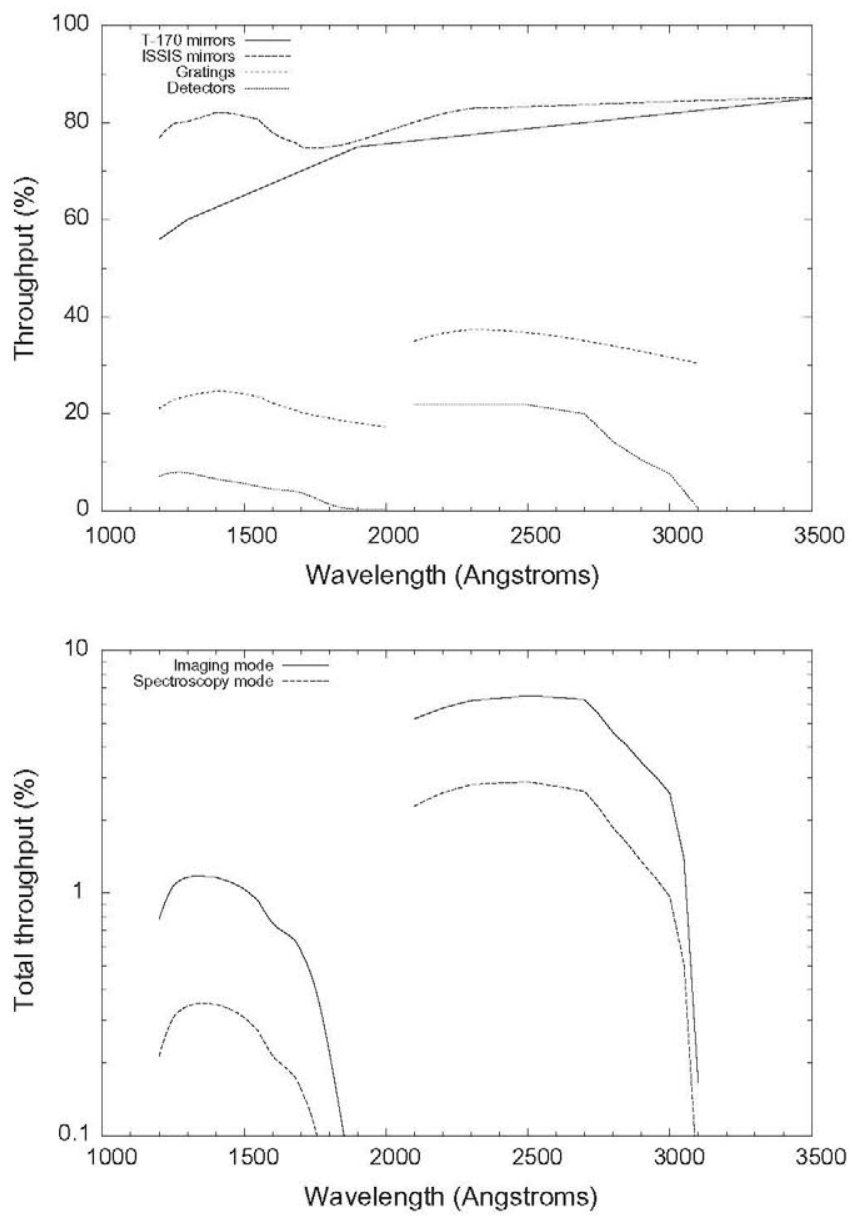

Fig. 4. Top: Sensitivity curves of the optical components for ISSIS: T-170 mirrors, ISSIS internal mirrors, gratings and detectors. Bottom: total throughput of the system in the imaging and spectroscopy modes. Filters are not included.

- Mode: for each channel the spectroscopy or imaging mode are allowed, and the user can select the optical filters on the wheels.

- Input data: the input can be given as a continuum flux, setting its value, a spectral line (absorption or emission), or a predefined distribution uploaded by the user as an ASCII file. Other options are a blackbody distribution
Table 1

Characteristics and performance of the ISSIS instrument.

\begin{tabular}{lll}
\hline & FUV channel & NUV channel \\
\hline Spectral range & $1150-175 \AA$ & $1850-3200 \AA$ \\
FoV imaging & $70 \times 75 \operatorname{arcsec}^{2}$ & $70 \times 75 \operatorname{arcsec}^{2}$ \\
FoV spectroscopy & $36 \times 65 \operatorname{arcsec}^{2}$ & $31 \times 61 \operatorname{arcsec}^{2}$ \\
Pixel scale & $0.036 \operatorname{arcsec}$ & $0.036 \operatorname{arcsec}$ \\
Scale ratio & $<7 \%$ & $<7 \%$ \\
Number of reflections & 4 & 4 \\
Temporal resolution & $40 \mathrm{~ms}$ & $40 \mathrm{~ms}$ \\
Detector type & $\mathrm{CsI} \mathrm{MCP}$ & $\mathrm{CsTe} \mathrm{MCP}$ \\
Detector diameter & $40 \mathrm{~mm}$ & $40 \mathrm{~mm}$ \\
Peak throughput & $\sim 1300 \AA$ & $\sim 2500 \AA$ \\
Spectroscopy resolution & $\mathrm{R}=500$ & $\mathrm{R}=500$ \\
Spatial resolution & 0.11 arcsec & 0.11 arcsec \\
Detector format & $>2048 \times 2048$ pixels & $>2048 \times 2048$ pixels \\
$\quad$ (equivalent) & & \\
\hline
\end{tabular}

(by choosing the effective temperature and magnitude), or an ATLAS9 atmosphere model by Castelli and Kurucz (2004).

- Parameters: in this section the user gives the desired S/ $\mathrm{N}$, and obtains the exposure time from the calculations, or vice versa.

The output window shows the calculated $\mathrm{S} / \mathrm{N}$ or time, depending on the user's choice, and three plots showing the input spectral distribution, the system throughput (telescope + ISSIS optical system), and the final observed flux. The user is allowed to see the results as a table, either in ASCII or HTML format.

\section{Conclusions}

This article presents ISSIS design at the end of Phase B. The instrument will be a versatile tool to analyse the UV sky that complements the other instruments onboard the WSO-UV space telescope. ISSIS flight model is due to be deliver to the Russian Federal Space Agency (Roscosmos) in 2015 for final integration and assembly. The WSO-UV launch is foreseen for 2017.
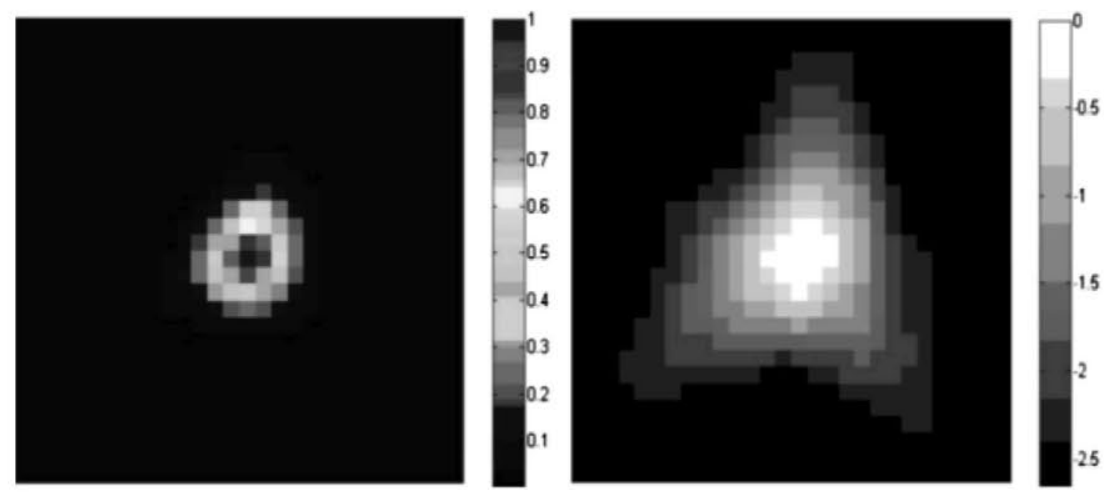

Fig. 5. A simulation of the PSF for the FUV channel in imaging mode in linear (left) and logarithmic (right) scales. 


\section{Exposure Time Calculator}

ISSIS - WSO-UV mission

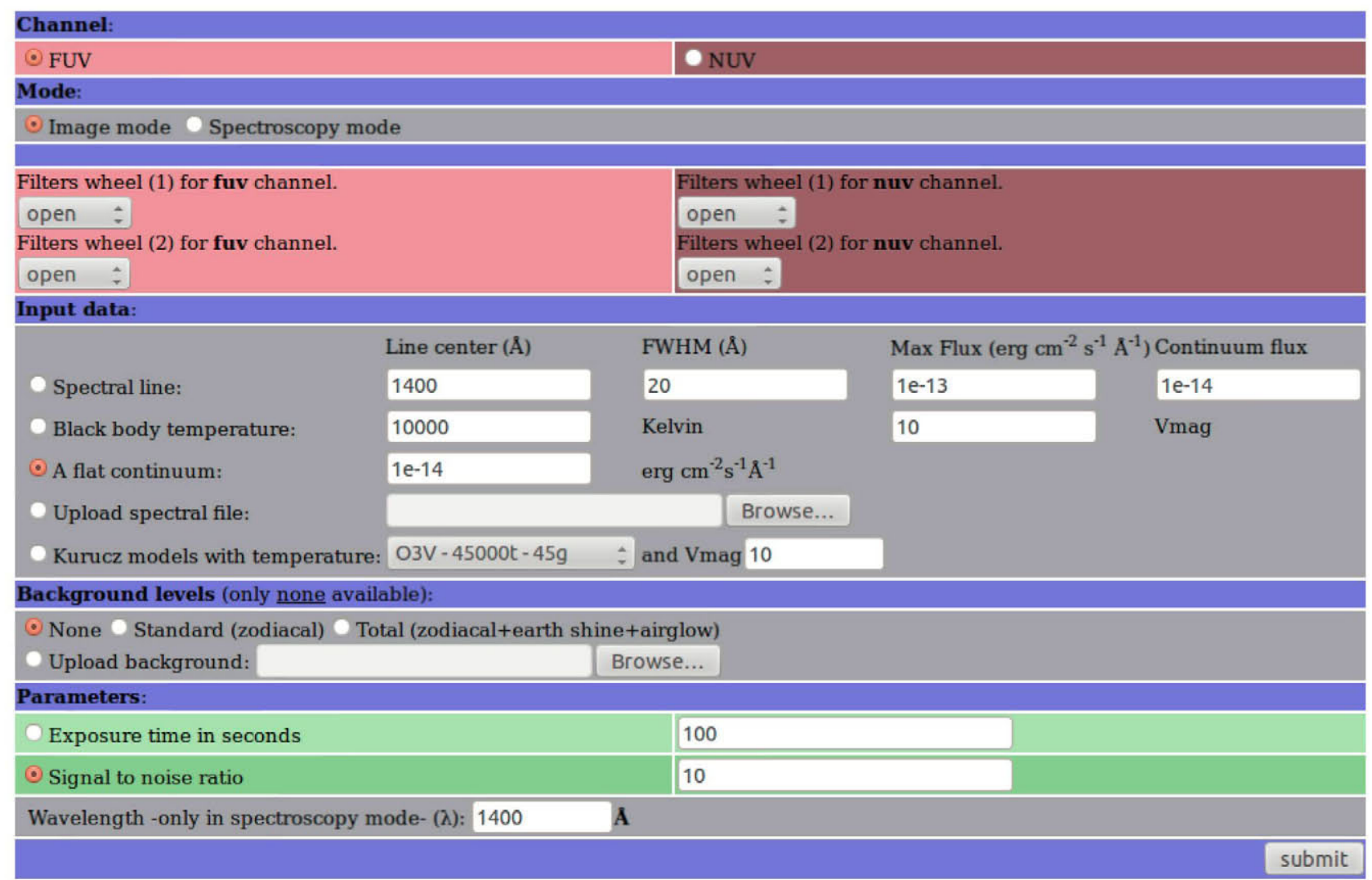

Fig. 6. Graphic interface of the ISSIS exposure time calculator.

\section{Acknowledgements}

ISSIS development is being funded by Ministry of Industry, Tourism and Commerce of Spain. We acknowledge the Spanish Science Team for their support in this project. We also would like to thank to the referees for their detailed revision of this manuscript. The science team at Universidad Complutense de Madrid acknowledges the financial support of the Ministry of Economy and Competitivity through Grants AYA2008-06423-C03-01 and AYA2011-29754-C03-C01.

\section{References}

Bianchi, L., Herald, J., Efremova, B., et al. GALEX catalogs of UV sources: statistical properties and sample science applications: hot white dwarfs in the Milky Way. Ap\&SS 335, 161-169, 2011.

Blay, P., Ribó, M., Negueruela, I. Very peculiar wind from $B D+53^{\circ} 2790$, the optical counterpart to 4U 2206+54. Ap\&SS 320, 145-148, 2009.

Caballero, J.A., Martín, E.L., Zapatero Osorio, M.R., et al. S Ori J053825.4-024241: a classical T Tauri-like object at the substellar boundary. A\&A 445, 143-153, 2006.

Castelli, F., Kurucz, R.L. New Grids of ATLAS9 Model Atmospheres, in: Piskunov, N., et al. (Eds.), Proceedings of the IAU Symp. No 210; Modelling of Stellar Atmospheres, poster A20 (CD-ROM publication), 2003. Available from: <arXiv:astro-ph/0405087>, 2004.
Coffey, D., Bacciotti, F., Woitas, J., et al. Rotation of jets from T-Tauri stars: new clues from HST/STIS observations. Ap\&SS 292, 553-558, 2004.

Gómez de Castro, A.I., Robles, A. Herbig-Haro objects. ESA SP-1237, 1999.

Gómez de Castro, A.I., Wamsteker, W., Barstow, M., et al. Fundamental problems in astrophysics. Ap\&SS 303, 133-145, 2006.

Gómez de Castro, A.I., Maíz-Apellániz, J., Rodríguez, P., et al. The imaging and slitless spectroscopy instrument for surveys (ISSIS) for the world space observatory-ultraviolet (WSO-UV). Ap\&SS 335, 283 289, 2011.

Gómez de Castro, A.I., Marcos-Arenal, P. Extended magnetospheres in pre-main-sequence evolution: from $\mathrm{T}$ Tauri stars to the Brown Dwarf limit. ApJ 749, id. 190, 2012.

Gómez de Castro, A.I., Sánchez Doreste, N., Sestito, P., et al. Scientific requirements for the Imaging and Slitless Spectroscopy Instrument for Surveys (ISSIS) for the World Space Observatory-Ultraviolet (WSOUV), Editorial Complutense, Madrid, 2012.

Gómez de Castro, A.I., Sánchez, N., Sestito, P., et al. ISSIS: the imaging and slitless spectroscopy instrument for surveys in the World Space Observatory-Ultraviolet telescope. Proceedings of the SPIE 8443, id. $84432 \mathrm{~W}, 2012 \mathrm{~b}$.

Lecavelier des Etangs, A., Vidal-Madjar, A., McConnell, J.C., et al. Atmospheric escape from hot Jupiters. A\&A 418, L1-L4, 2004.

Lecavelier des Etangs, A., Ehrenreich, D., Vidal-Madjar, A., et al. Evaporation of the planet HD 189733 b observed in H I Lyman- $\alpha$. A\&A 514, id. A72, 2010.

García, M., Herrero, A., Najarro, F. WSO and the winds of massive stars: the gate to the metal-poor Local Universe. Ap\&SS 335, 91-96, 2011. 
Goicoechea, L.J., Shalyapin, V.N. Time delays in the gravitationally lensed quasar H1413+117 (Cloverleaf). ApJ 708, 995-1001, 2010.

Guerrero, M.A., Ramos-Larios, G., Massa, D. A FUSE view of winds from the central stars of planetary nebulae. PASA 27, 210-213, 2010.

Guerrero, M.A., Ruiz, N., Hamann, W.-R., et al. Rebirth of X-Ray emission from the born-again planetary nebula A30. ApJ 755, id. 129, 2012.

Martin, C., Barlow, T., Barnhart, W., et al. The Galaxy Evolution Explorer. Proceedings of the SPIE 4854, 336-350, 2003.
Murthy, J., Henry, R.C., Sujatha, N.V. Mapping the diffuse Ultraviolet Sky with the Galaxy Evolution Explorer. ApJ 724, 1389-1395, 2010.

Sachkov, M. UV observations of $\mathrm{sdB}$ stars and prospects of WSO-UV mission for such studies. A\&SS 329, 261-266, 2010.

Shustov, B., Sachkov, M., Gómez de Castro, A.I., et al. World space observatory-ultraviolet among UV missions of the coming years. Ap\&SS 335, 273-282, 2011.

Sujatha, N.V., Murthy, J., Karnataki, A., et al. GALEX observations of diffuse UV radiation at high spatial resolution from the Sandage Nebulosity. ApJ 692, 1333-1338, 2009. 